\title{
Fuzzy based Distributed Cluster Formation and Route Construction in Wireless Sensor Networks
}

\author{
Melaku Tamene \\ Dept. of CS \& SE, AUCE \\ Andhra University \\ Visakhapatnam, INDIA
}

\author{
Kuda Nageswara Rao \\ Dept of CS \& SE, AUCE \\ Andhra University \\ Visakhapatnam, INDIA
}

\begin{abstract}
The efficiency of cluster formation has a sound effect on limiting battery lifetime in cluster based wireless sensor networks. Fuzzy based decision support system enables node to make evaluation and pass a soft decision on the state of being configured as normal or cluster leader without the presence of precise information about different clustering parameters. In this paper, a distributed fuzzy based cluster formation is presented in which the set of appropriate cluster heads are elected to setup clusters and optimal routes toward the base station are constructed based on the candidate gateway nodes. Initially, the base station partitions the network into different tiers and then nodes define themselves to the corresponding tier. The minimum distance from the border of corresponding tier and energy level limit the selection probability of node to become cluster head or gateway node. The fuzzy logic toolbox is developed in $\mathrm{C}++$ and integrated with OMNeT++ simulation platform to implement the protocol and experimental results reveal that the proposed protocol prolongs the network lifetime compared to LEACH-C and CHEF protocols.
\end{abstract}

\section{Keywords}

Cluster head election, fuzzy based cluster formation, network lifetime, wireless sensor networks.

\section{INTRODUCTION}

Nowadays, microsensors are integrated with microprocessor and radio to sense, compute and communicate with other nearby or remote devices. Wireless sensor networks (WSNs) are made up of miniature size sensor nodes that are capable of sensing any physical phenomenon and reporting data to the sink in coordinated manner [1]. The basic hardware components of senor node include sensors, signal conditioning unit, microcontroller, radio, power source and sometimes locomotive units are also integrated to allow mobility features [2-4]. As smart sensors can be kept all around to monitor the physical phenomena in our day to day activities, the application of sensor networks is enormously vast [5-6]. In medical applications, the physical and biological sensors can be either wore or implanted inside human body to continuously monitor patients with long lasting diseases. In military, sensor networks assist the armed forces to have battlefield surveillance through reporting the enemy vehicle location or movement analysis, sniper detection and location estimation. In precision agriculture, sensor networks are used to increase productivity by regulating the amount of fertilizers or water in the region based on measuring the soil moisture and air temperature. Sensor networks can also be applied for smoke detection in industry, water or gas pipeline monitoring, damage monitoring in building and habitant control. As the technology advances, even home appliances such as microwave ovens and refrigerators are also integrated with smart sensors so that the user shall be able to control them remotely.

While sensor networks have vast applications in real world, they are challenged with limited power supply [7-8]. Considering that sensor nodes have only onboard power supply and due to their placement in hostile environment to make battery replenishment, hardware and software architectures in WSNs shall focus on efficient use of energy. Most of the energy expenditure in sensor node is linked with communication radio. Hence, low power radio designs and energy saving communication protocols development are the preferred solutions to elongate the functionality of sensors in WSNs. Dynamic change of network topology and self organization are other challenges in wireless sensor networks. As sensor nodes are not manually inspected after random placement in the sensor field, they are expected to organize themselves so as to adapt with frequent change of network topology due to failure or mobility [9]. Full coverage and connectivity in the desired sensor field can be achieved if nodes are deployed with high density. Since large scale deployment causes overlapping of sensing coverage, most of sensors reading are similar. Data redundancy can be minimized if nodes are able to organize themselves to form clusters and then compress the correlated data into a single packet before forwarding to the base station. Cluster formation not only reduces the volume of data sent to the sink but also minimizes the channel contention [10-12]. Cluster formation incorporates election of cluster heads ( $\mathrm{CHs})$ and association with their corresponding cluster members. It is proved that routing protocols based on clustering provide better network lifetime compared to flat routing protocols.

Traditional computing methods need complex mathematical modeling and tedious computational effort to come up with optimal solution in design of communication protocols that are required to adapt with dynamic nature of WSNs. Nowadays, WSNs are embraced with computational intelligence (CI) that nodes can have advanced features like perception of the environment, reasoning, knowledge, learning and decision making. The evolutionary computation, artificial neural network and fuzzy logic are used to solve different optimization problems in WSNs such as energy aware routing, optimal deployment and localization. In this paper, a new clustering protocol named by FDCR (Fuzzy based Distributed Cluster Formation and Route Construction) is introduced which selects the right set of cluster heads based on fuzzy logic and route towards the sink is built through gateway nodes. The protocol is implemented using OMNeT++ simulation tool and the result verifies that FDCR extends the network lifetime compared to the prominent LEACH-C and CHEF protocols. 
The rest of the paper is organized as follows. Section 2 describes the literature survey of famous routing protocols in cluster based networks. Section 3 explains the system model. Proposed protocol design and analysis is described in section 4. Section 5 explains the simulation results and section 6 concludes the paper.

\section{RELATED WORK}

One of the earliest cluster based algorithms is low energy adaptive clustering hierarchy (LEACH) protocol [13]. Nodes arbitrarily take any number between 0 and 1 and decide to act as cluster head if the number is less than the threshold value $T(n)$. At the end of cluster setup phase, the state of each node is either cluster member or leader (cluster head) in LEACH protocol.

$$
T(n)= \begin{cases}\frac{p}{1-p\left(r \bmod \frac{1}{p}\right)} & , \text { if } n \varepsilon g \\ 0 & \text {, otherwise }\end{cases}
$$

Where $p, r$ and $g$ are the preferred percentage of cluster heads, current round number and the set of nodes that are not selected as cluster head in the previous $1 / p$ rounds respectively. The cluster heads broadcast their state using nonpersistent carrier sense multiple access (CSMA) MAC protocol and the regular nodes choose one of least cost cluster heads based on RSSI (received signal strength indicator) value. The cluster heads identify their members upon the reception of join request message from regular nodes. The cluster members send their frames to the respective cluster heads based on pre assigned TDMA slot. In steady state data transmission phase, the cluster head aggregates the data collected from members and then send to the base station using spreading codes and CSMA. Sensing the channel before sending the actual data reduces interference. Regular nodes take their turn to compete for being elected as cluster head in subsequent rounds to distribute burden imposed on cluster heads. LEACH protocol does not consider the residual energy of nodes in cluster formation and the number of cluster heads stochastically varies in each round. besides, the distribution of cluster heads in LEACH is as such non-uniform that too much of cluster heads may be confined in one region while there is a few or no cluster head in the other portion of sensor field.

The work in [14] introduces centralized LEACH (LEACH-C) protocol. Unlike LEACH, instead of building clusters independently in distributed manner, the base station centrally configures the cluster heads, defines the associated cluster members and then it will broadcast the information back to nodes in each round. Then, nodes identify their TDMA slot and switch off their radio (sleep mode) accordingly to save power till the time for data transmission has reached. In each cluster setup phase, nodes are supposed to send location (using integrated GPS receiver) and energy information to the base station. The base station computes the average energy of nodes and by using the set of nodes whose energy exceeds the average as possible set of cluster heads, it finds the optimal clusters using simulated annealing algorithm. The algorithm attempts to reduce intra-cluster communication cost through minimizing the squared distance between cluster members and the corresponding cluster heads. The steady state data transmission phase is similar to LEACH.

Energy efficient unequal clustering (EEUC) protocol is proposed in [15]. In multi-hop data transmission, the sensor field at the closest distance to the base station is hot spot area where any packet meant for base station goes through it and hence the expected energy dissipation of nodes in this region is very high compared to others. The fast rate of energy dissipation in hot spot area is accompanied with the problem of energy hole near to the base station. The energy cavity problem in sensor nodes located near to the base station is soon accompanied with loss of control about the physical events appeared in some sections of WSN field. The EEUC protocol tries to reduce the problem of energy hole by managing the size of clusters built in different sections of sensor field. Nodes in hot spot area are more burdened with inter-cluster communication and hence they shall to minimize intra-cluster communication cost by keeping smaller cluster size. The work in [16] extends EEUC protocol using fuzzy set theory in which nodes determine their competition range based on distance from the base station and current energy level.

The authors in [17] present a hybrid, energy efficient, distributed clustering (HEED) protocol. The remaining energy of node is primarily considered to elect the set of temporary cluster heads. In HEED, nodes begin with identification of neighbors and setting of their cluster head probability chpro . To terminate execution of HEED within fixed number of iterations, the cluster head probability of each node is not permitted to fall below pre assigned threshold value. Node which is not capable of hearing any temporary or final cluster head message is assumed uncovered and candidates itself to become cluster head with probability chpro. The cluster head probability of each node is doubled in between iterations and the one whose energy is higher compared to others terminates the execution soon. If node does not hear any final cluster head message at the end of execution, it will declare itself as final cluster head. A regular node which resides in the transmission range of more than one cluster head will break the competition ties by joining the one with minimum cost. The cost metric may be function of node degree, proximity to neighbors or inverse of node degree depending on the requirement of clusters. HEED provides good distribution of cluster heads since it is less probable for two cluster heads to reside within the transmission range of each other.

Most of the research papers give more weight on formation of clusters based on the current energy level of nodes and very little has been done on consideration of the expected residual energy after cluster formation. In [18], the authors propose a fuzzy based clustering approach by taking energy prediction into account. At the beginning of every cluster formation phase, nodes predict their residual energy upon evaluation of the expected energy consumption of being cluster head. The current energy level and expected residual energy are input parameters of fuzzy based decision system by which nodes evaluate their chance to act as cluster head.

The paper in [19] describes cluster head election mechanism using fuzzy logic (CHEF). The fuzzy logic is used to evaluate the chance of each node to become cluster head. The fuzzy system computes the selection probability of each node based on the local distance and current energy level. Nodes initially made neighbor discovery and the distance is measured using received radio signal strength. In CHEF, the local distance of a node is defined as the sum of the distance of itself from its neighbor nodes. Initial set of candidate cluster heads are elected probabilistically and designation as final cluster heads relies on comparison of their chance with those nodes that reside within the competition range. The relative strength of node to win the competition depends on the magnitude of selection probability. 
In [20], an improved fuzzy based unequal clustering algorithm is presented. The clustering parameters used in the algorithm are distance to the base station, energy and neighbor density. Using the fuzzy system to work on the given parameters, the algorithm determines the competition radius and the strength of each node to act as cluster head. The use of variable cluster radius in cluster formation reduces hot spot problem in multihop data transmission. After the cluster head receives data from its respective cluster members, it will make neighbor discovery and the set of cluster heads nearer to the base station are saved in its neighbor list. Then, Ant Colony Optimization (ACO) algorithm is used to select next hop cluster head during inter-cluster data dissemination towards the base station.

\section{SYSTEM MODEL}

\subsection{Modeling Energy Consumption of Sensor Node}

Accurate modeling of energy consumption in sensor node helps the network designers to have a good estimate of system lifetime. The source of energy consumption in sensor node is related with sensing, radio transmission and reception, microcontroller processing, operational state transition and sensor logging. The simplified first order radio model has been adopted to estimate the energy consumption in radio communication [21]. The energy expenditure in the radio is associated with dissipation in transmitter and receiver circuits. The receiver dissipates energy for running the receiver electronics while the transmitter expends for running the transmitter electronics and power amplifier. The free space ( $d^{2}$ power loss) and multipath fading ( $d^{4}$ power loss) radio wave propagation models are used upon the separation of transmitting and receiving node [22]. Let the energy consumed for transmitting $k$ bits of data to a distance $d$ and receive the same size of data be $\operatorname{ETx}(k, d)$ and $\operatorname{ERx}(k)$ respectively. Then, energy dissipation in radio hardware can be written as follows.

$$
\begin{gathered}
\operatorname{ETx}(k, d)=\left\{\begin{array}{l}
k \text { Eelc }+k \text { efs } d^{2}, d<d o \\
k \text { Eelc }+k \text { emp } d^{4}, d \geq d o
\end{array}\right. \\
E R x(k)=k \text { Eelc } \\
d o=\sqrt{\frac{e f s}{e m p}}
\end{gathered}
$$

The symbol Eelc represents the energy dissipation per bit in transmitter or receiver electronics. efs, emp are energy dissipation factors of amplification for the corresponding channel models. Generally, for experiments in this paper, the assumed energy dissipations are due to the energy consumption of microcontroller for data processing in cluster heads and the power loss for communications in radio hardware.

\subsection{Network Model and Assumptions}

The topology of wireless sensor networks is represented as a graph $G=(V, E)$ where each vertex $v \in V$ in the graph indicates sensor node and an edge $(u, v) \in E$ represents the wireless communication link between nodes. Two nodes are assumed to be neighbors if the Euclidean distance between them is less than their transmission radius. Sensor nodes are not movable after deployment and they have the same computing power, memory size and sensing abilities.
Consider that nodes can locate their position whenever required and they have variable transmission power levels. Consider the available discrete transmission power levels of each node are denoted in a finite set $S \ell$. If node intends to transmit over a distance $d i j$, then it will make a choice on the optimum transmission power level (Topt) among values in the set $(P \ell)$ as follows.

$$
\text { Topt }^{i j}=\arg \min P \ell \in S \ell\{\operatorname{ETx}(k, d)\}
$$

The wireless channel is assumed to be symmetric in the model. Battery is the only source of energy in sensor nodes and any other energy scavenging (harvesting) features is not incorporated in the system.

\section{PROPOSED PROTOCOL DESIGN}

Suppose $(0,0),(x m, 0),(0, y m),(x m, y m)$ define the coordinates of corner of rectangular WSN field and the base station is positioned exterior to deployment region at $\left(x_{o}, y_{o}\right)$ as shown in Figure 1. Let $r 0, r 1, \ldots, r n$ represent the various transmission range of base station with respect to its available discrete transmission power levels. Initially, the base station partitions the network into different tiers through varying its transmission power and nodes will then associate themselves to the corresponding tier. Nodes define their tier in accordance with their distance from the base station. For instance, nodes within the range of minimum transmission power of base station are labeled as first tier nodes. So, for multiple tier networks, nodes define their tier as first, second, third and the like upon hearing the announcement from the base station. The base station divides the network into a number of tiers depending on the density of nodes in WSN field. The distance of each sensor node from the border of corresponding tier and battery power level mutually decide the class of node as regular, gateway or cluster head. Suppose node is located at $\left(x^{\prime}, y^{\prime}\right)$ in WSN field, the shortest distance of a node from the border of its corresponding tier $(d s)$ can be formulated as follows.

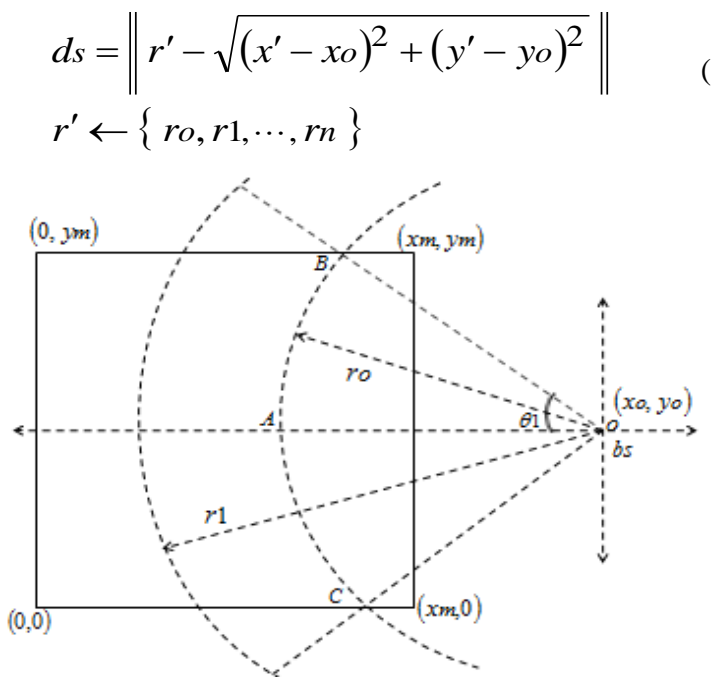

Figure 1: Topology map of WSN field

Consider two nodes with the same energy located at the same distance from the border of their corresponding tier, these two nodes are equally capable to be elected as cluster head in the proposed model even if they are actually at different distance from the base station. This scenario enables the network to have good distribution of cluster heads. Otherwise, those 
nodes very near to the base station are usually favored in cluster head election which aggravates the rate of depletion of energy in this region and those nodes located at far distance are either uncovered or they will be at least subjected to long distance transmission to forward their data.

The selection probability of nodes upon the given clustering parameters is computed based on fuzzy logic. The operation of protocol incorporates cluster formation and route construction phases. The gateway node election and route construction towards the base station are accomplished right after nomination of cluster heads and identification of the corresponding cluster members. Distance from the border of corresponding tier and remaining energy of node are the input parameters for FIS (fuzzy inference system) based chance computation as it is depicted in the Figure 2. The fuzzy set representing input and output linguistic variables are shown in Figure 3. Input fuzzy variables use Low, Medium and High linguistic values. Very Weak, Weak, Little Weak, Little Medium, Medium, Medium High, Little Strong, Strong and Very Strong linguistic values are used for output fuzzy variables. Low, High, Very Weak and Very Strong linguistic values are represented by trapezoidal membership function and the rest of linguistic values use triangular membership function. The fuzzy if-then mapping rules of fuzzy inference system are shown in Table 1 and Mamdani implication has been used for interpretation of rules. Center of gravity defuzzification technique is used to accomplish the transformation of fuzzy output to crisp value.

$z^{*}=\frac{\sum \mu_{a}(z) \cdot z}{\sum \mu_{a}(z)}$

Where the parameter $z^{*}$ represents the defuzzified output, $\mu(z)$ is the membership function after aggregation and $z$ is the output variable.

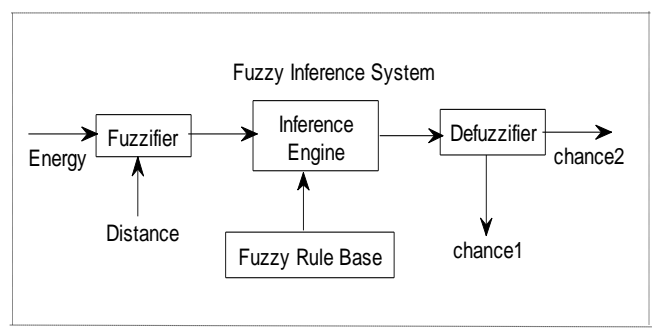

Figure 2: Architecture of FIS based chance computation

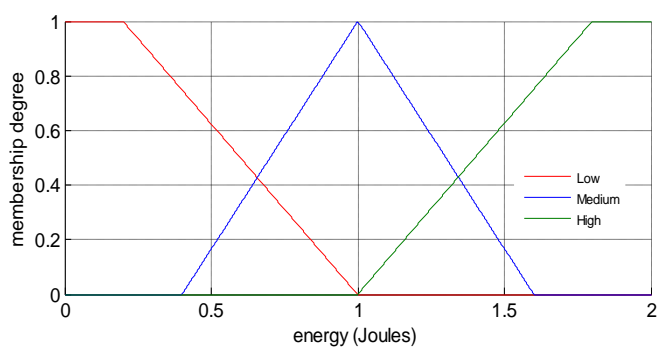

Figure 3: ( $a$ ) Fuzzy set for remaining energy

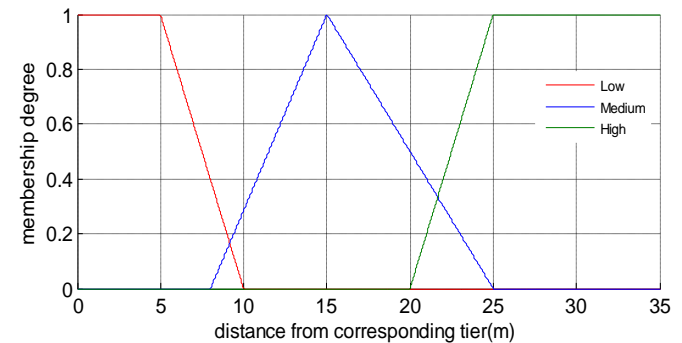

Figure 3: $(b)$ Fuzzy set for distance

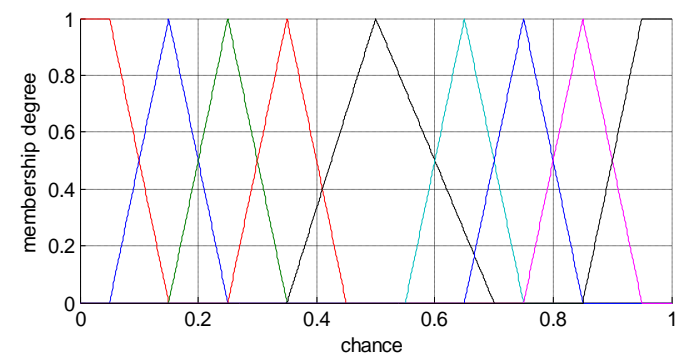

Figure 3: ( $c$ ) Fuzzy set for selection probability

Table 1. Fuzzy if-then rules

\begin{tabular}{c|c|c|c}
\hline Energy & Distance & Chance $(\mathbf{C H})$ & Chance (gateway) \\
\hline Low & Low & Very Weak & Little Weak \\
Low & Medium & Weak & Weak \\
Low & High & Little Weak & Very Weak \\
Medium & Low & Little Medium & Medium High \\
Medium & Medium & Medium & Medium \\
Medium & High & Medium high & Little Medium \\
High & Low & Little Strong & Very Strong \\
High & Medium & Strong & Strong \\
High & High & Very Strong & Little Strong \\
\hline
\end{tabular}

\subsection{Distributed Cluster Formation Algorithm}

At the beginning of every cluster construction phase, each sensor node determines the chance of being cluster head $(\mu 1)$ and gateway node ( $\mu 2)$ using fuzzy logic. Let the symbol $T$ represents the desired percentage of cluster heads. Initially, each node generates a random number between 0 and 1 and assigns itself as temporary cluster head if the generated number is less than the threshold value $(T)$. The pseudocode for cluster head election process is shown in Algorithm-1. Assigning initial set of temporary cluster heads upon probabilistic nature is aimed for limiting control packets overhead and competition of nodes to qualify for final cluster head election process relies on fuzzy computed chance value. The temporary cluster head announces TempoCHMessage within competition range embedding $\mu 1$ in its advertisement and set a timer to evaluate its chance compared to other temporary cluster heads within the radio range. Of the temporary cluster heads, the one whose $\mu 1$ value outweighs other nearby temporary heads broadcasts FinalCHMessage. Nodes which are not initially assigned as temporary cluster head or quit from election process due to lower $\mu 1$ value append $\mu 2$ in their JoinCHMessage advertisement that is meant for the nearest final cluster head. 


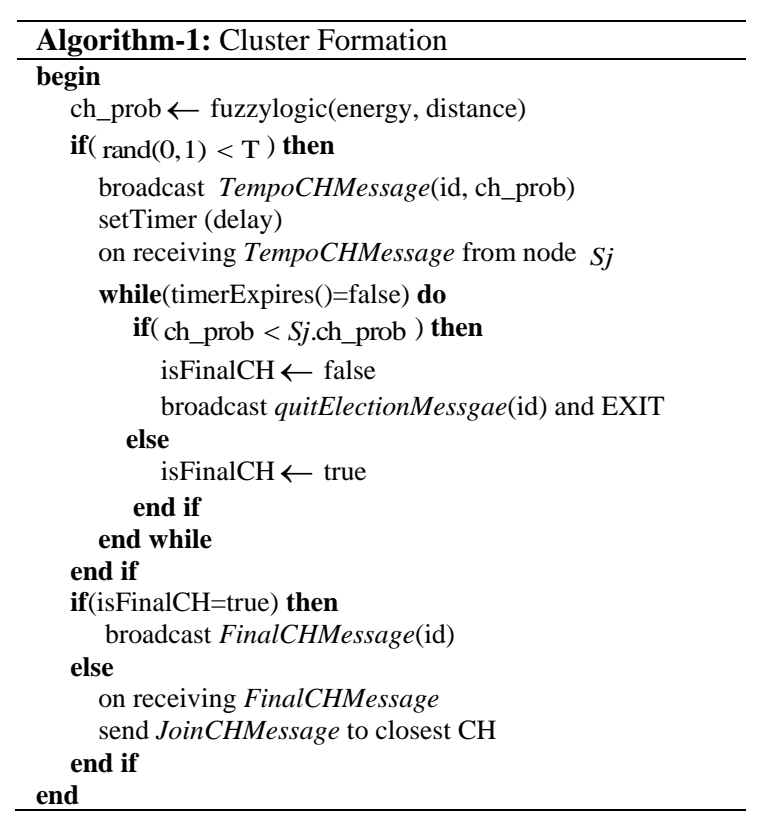

\subsection{Gateway Node Election and Route Construction}

The final cluster heads are responsible to prospect initial set of gateway nodes to reduce communication overhead imposed in gateway node election. The pseudocode for gateway node election and route construction is depicted in Algorithm-2. On the reception of JoinCHMessage, the final cluster head assigns one of its children having maximum $\mu 2$ value as a candidate gateway node. The cluster head selects one of its children randomly if they have same $\mu 2$ value. The cluster head broadcasts id of the candidate gateway node and time schedule to transfer data. Then, regular cluster members enter sleep mode to save their power while gateway node sets a timer to announce its state. The reason for nodes except cluster heads and gateway nodes to switch off their radio at this stage is that they do not have any particular contribution with route construction. Consider the neighbors set of node $S j$ is represented as $N(S j)$. Let $d S j$ be the distance of node $S j$ to base station and $d$ next be the distance from one of neighbors of node $S j$ to the base station. Upon the reception of GatewayNodeMessage, the cluster heads and gateway nodes maintain a record of downstream nodes (forward candidate set) from the set of their neighbors and the advertisement message from any node in the upstream set will be dropped in route construction. Nodes in the neighbor set can be classified to upstream $(S u)$ and downstream $(S d)$ sets based on the following equation.

$$
\begin{gathered}
S d \leftarrow\{v: v \in N(S j) \text { and } d S j-\text { dnext } \geq 0\} \\
S u \leftarrow\{v: v \in N(S j) \text { and } d S j-\text { dnext }<0\}
\end{gathered}
$$

Nodes in the forward candidate set $(S c$ ) are evaluated based on the magnitude of received signal strength and the one with minimum cost will be assigned as gateway node during route construction. Consider the vector containing the magnitude of received signal strength of forward candidates is denoted as $\mathbf{f} r$, the selection of gateway node ( $\mathrm{Sg}$ ) can be accomplished in the following manner.

$$
\mathbf{f} r=[f r 1, f r 2, \ldots, f r n]^{T} \mathbf{f} r \in R^{\|S c\|}
$$

$$
S g=\arg \min _{s \in S d}\{\mathbf{f} r\}
$$

If members do not exist in the forward candidate set, the base station will be assigned as the default gateway. Suppose $M$ number of gateway nodes is selected from source to destination in route construction, then the set containing list of gateway nodes can be represented as follows.

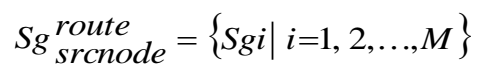

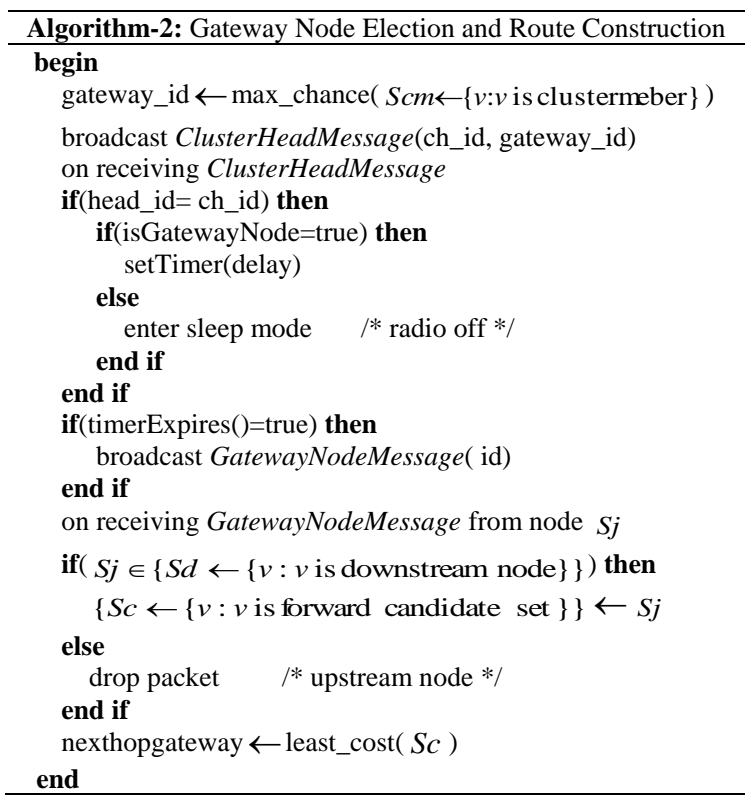

\subsection{Protocol Operation}

After the completion of cluster setup and route construction, cluster members send data packets to their corresponding cluster heads in accordance with the pre-assigned TDMA time slots. Then, the cluster head works on compression of highly correlated data and send the aggregated data destined for base station through gateway nodes. The steady state data transmission phase is a lot like LEACH protocol and hence the detail is omitted here.

\section{PROTOCOL PERFORMANCE EVALUATION}

\subsection{Simulation Environment}

Setup of the actual hardware components to build large scale sensor networks is often expensive in terms of time, cost and complexity to test and analyze new protocols. Having considered that a large number of system configurations can be controlled in the simulation models, simulators are used as a powerful and flexible tool in designing networks. Marvelous conclusions that have practical importance can be drawn from simulators as far as the actual definitions of system are included in the simulation model. The experiment is conducted using objective modular network testbed (OMNeT) simulation platform. OMNeT provides a component based architecture for models where the components are programmed in $\mathrm{C}++$ and they can be connected to each other or come together to form compound modules using high level network description language (NED) [23]. The fuzzy logic toolbox is developed in $\mathrm{C}++$ and integrated with OMNeT ++ simulation environment. A total of 100 sensor nodes are 
uniformly distributed in $100 m \times 100 m$ WSN area. The configuration parameters used in the simulation are initial energy $=2 \mathrm{~J}$, base station location $=(175,50) \mathrm{m}$, control packet size $=25$ bytes, data packet size $=500$ bytes, Eelc $=50 \mathrm{~nJ} / \mathrm{bit}$, energy loss for data aggregation $(E d a)=5 \mathrm{~nJ} / \mathrm{bit} / \mathrm{signal}$, efs $=10 \mathrm{pJ} / \mathrm{bit} / \mathrm{m}^{2}$, emp $=0.0013 \mathrm{pJ} / \mathrm{bit} / \mathrm{m}^{4}$, competition radius $=25$ $m$ and TDMA frames per round $=6$.

\subsection{Simulation Results and Analysis}

The performance of protocols in WSNs is primary measured by network lifetime. The definition of network lifetime is rather application dependant but the most generic definition is the time elapsed till the network is considered non-functional. Actually, the perception of non-functionality varies with application requirement. From this point of view, the network lifetime can be generally taken as the time elapsed till first node dies (FND), the time till some percentage of nodes die (PND), the time till last node dies (LND) or the time till there is loss of coverage and connectivity in some portion of sensor field. Having considered that the network is assumed functional as far as significant proportion of nodes are working in most of network applications and due to the fact that adjacent nodes can capture identical or at least similar data in large scale sensors deployment whenever a node is subjected to failure, the network lifetime has been defined as the time elapsed till 50\% of nodes die (HND). In Figure 4, the number of operational nodes per each round is examined and the simulation result shows that FDCR protocol improves the network lifetime by $9.62 \%$ and $6.51 \%$ compared to LEACH-C and CHEF protocols respectively.

Load balance among nodes is another important metric in WSNs. To examine the status of homogeneity in energy consumption, the standard deviation of residual energy of nodes per each data gathering round is computed. The simulation result in Figure 5 justifies that FDCR has relatively uniform energy consumption among nodes than LEACH-C and CHEF protocols. The average energy consumption of cluster heads is also evaluated in the experiment as it is shown in Figure 6. The average of data points (energy consumption of cluster heads) are taken in each consecutive 25 rounds and the simulation result proves that FDCR protocol has lower energy consumption than its counterparts.

Considering that there is strong correlation of data packets in WSNs, the available packets within the cluster shall be processed before transmission to the base station. So, the volume of packets received at the base station after data aggregation in cluster heads (effective data packets) has been considered in the experiment. Figure 7 shows the number of working nodes per amount of effective data packets received at the base station for each protocol. FDCR delivers more effective data packets than LEACH-C protocol for the same number of node deaths. FDCR does the same compared to CHEF until $83 \%$ of nodes are alive below which CHEF protocol dominates FDCR.

\section{CONCLUSION}

Frequent topology changes because of either mobility or failure and limited power supply are inherent problems in wireless sensor networks. Coordinated cluster formation is an optimal routing solution for energy constrained WSNs. Energy efficiency is the critical design issue in cluster formation. In this paper, a distributed cluster formation is presented where the right set of cluster heads are elected based on fuzzy logic to build clusters and route towards the base station is constructed using gateway nodes. Experimental studies prove that the proposed protocol performs better and improves the network lifetime compared LEACH-C and CHEF protocols. FDCR protocol considers static nodes after deployment. Having considered that there are some applications in WSNs that highly get advantage from mobility features, part of the future work shall focus on design of cluster based protocol for mobile wireless sensor networks.

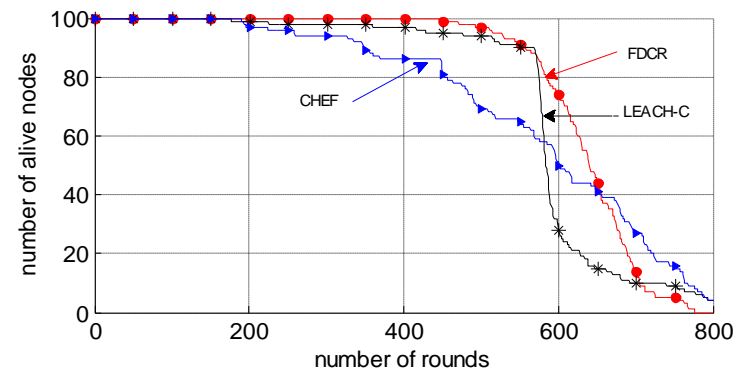

Figure 4: Number of alive nodes per simulation rounds

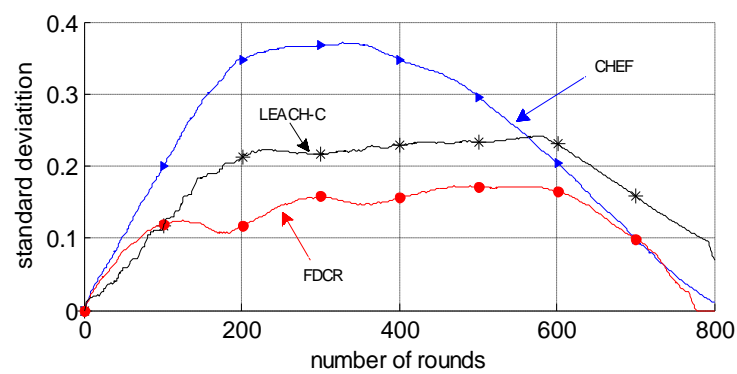

Figure 5: The standard deviation of energy among nodes

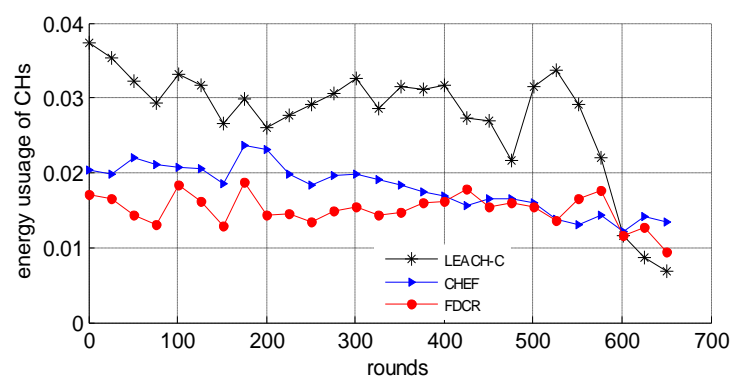

Figure 6: Average energy consumption of cluster heads

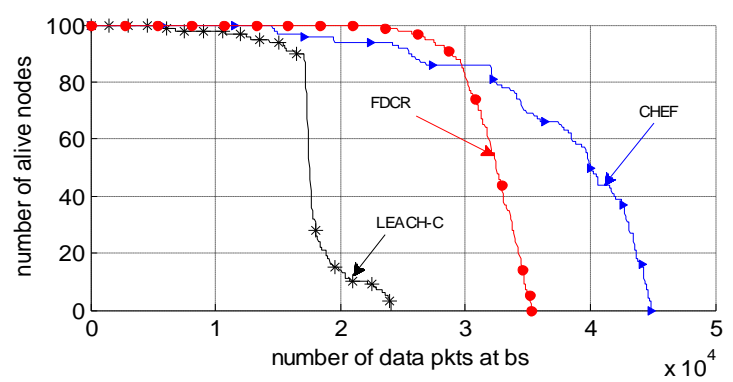

Figure 7: Alive nodes per data packets sent to base station

\section{REFERENCES}

[1] Akyildiz, I.F., Weilian, S., Sankarasubramaniam, Y. and Cayirci, E. 2002. A survey on sensor networks. IEEE Communications Megazine, vol.40, pp.102-114.

[2] Asada, G., Dong, M., Lin, T.S., Newberg, F., Pottie, G., Kaiser, W.J. and Marcy, H.O. 1998. Wireless integrated network sensors: Low power systems on a chip. In 
Proceedings of the 24th European Solid-State Circuits Conference (ESSCIRC), pp. 9-16.

[3] Sohrabi, K., Ailawadhi, V., Gao, J. and Pottie, G.J. 2000. Protocols for self-organization of a wireless sensor network. IEEE Personal Communications Magazine, vol. 7, no. 5, pp. 16-27.

[4] Anastasi, G., Conti, M., Francesco, M.D. and Passarella, A. 2009. Energy conservation in wireless sensor networks: A survey. Ad Hoc Networks, vol.7, no.7, pp. 537-568.

[5] Martinez, D., Blanes, F., Simo, J. and Crespo, A. 2008. Wireless sensor and actuator networks: characterization and case study for confined spaces health care applications. In Proceedings of International Conference on Computer Science and Information Technology (IMCSIT), pp.687-693.

[6] Reinisch, C., kastner, W., Neugschwandtner, G. and Granzer, W. 2007. Wireless Technologies in home and building automation. In Proceedings of IEEE International Conference on Industrial Informatics, vol.1, pp.93-98.

[7] Ephremides, A. 2002. Energy concerns in wireless networks. IEEE Wireless Communications Magazine, vol.9, no. 4 , pp. $48-59$.

[8] Borges, L.M., Velez, F.J and Lebres, A.S. 2014. A survey on the characterization and classification of wireless sensor networks applications. IEEE Communications Surveys Tutorials, vol. 16, no. 14, pp. 1860 - 1890 .

[9] Gungor, V.C. and Hancke, G.P. 2009. Industrial wireless sensor networks: Challenges, design principles, and technical approaches. IEEE Transactions on Industrial Electronics, vol. 56, no. 10, pp. 4258-4265.

[10] Abbasi, A.A. and Younis, M. 2007. A survey on clustering algorithms for wireless sensor networks. Computer Communications, vol.30, pp.2826-2841.

[11] Ai-hua, Q. 2012. A Research of clustering algorithms and its improvements in wireless sensor networks. In Proceedings of IEEE International Conference on Computer Science and Network Technology (ICCSNT), pp.1835-1838.

[12] Younis, O., Krunz, M. and Ramasubramanian, S. 2006. Node clustering in wireless sensor networks: Recent developments and deployment challenges. IEEE Network, vol. 20, no. 3, pp. 20-25.
[13] Heinzelman W., Chandrakasan, A. and Balakrishnan, H. 2000. Energy efficient communication protocol for wireless microsensor networks. In Proceedings of IEEE Hawaii International Conference on System Sciences, pp.3005-3014.

[14] Heinzelman W., Chandrakasan, A. and Balakrishnan, H. 2002. Application specific protocol architecture for wireless microsensor networks. IEEE Transactions on Wireless Communications, vol. 1, no. 4, pp. 660-670.

[15] Li, C., Ye, M., Chen, G. and Wu, J. 2005. An energyefficient unequal clustering mechanism for wireless sensor networks. In Proceedings of IEEE International Conference on Mobile Adhoc and Sensor Systems.

[16] Bagci, H. and Yazici, A. 2010. An energy aware fuzzy unequal clustering algorithm for wireless sensor networks. In Proceedings of IEEE International Conference on Fuzzy Systems (FUZZ), pp.1-8.

[17] Younis, O. and Fahmy, S. 2004. HEED: a hybrid energy efficient, distributed clustering approach for ad hoc sensor networks. IEEE Transactions on Mobile Computing, vol.3, No.4, pp.366-379.

[18] Lee, J. and Cheng, W. 2012. Fuzzy-logic-based clustering approach for Wireless sensor networks using energy Predication. IEEE Sensors Journal, vol. 12, no. 9, pp. $2891-2897$.

[19] Kim, J., Park, S., Han, Y. and Chung, T. 2008. CHEF: Cluster head election mechanism using fuzzy logic in wireless sensor networks. In Proceedings of 10th International Conference on Advanced Communication Technology, pp. 654-659.

[20] Bagci, H. and Yazici, A. 2011. An improved fuzzy unequal clustering algorithm for wireless sensor network. In Proceedings of International Conference on Communications and Networking in China (CHINACOM), pp.245-250.

[21] Smithgall, D. 1998. Toward the 60 gm wireless phone. In Proceedings of the 1998 Radio and Wireless Conference (RAWCON).

[22] Rappaport, T. 1996. Wireless Communications: Principles and Practice. Englewood Cliffs, NJ:PrenticeHall.

[23] OMNeT++ Network Simulator. [Online]. Available: http://www.omnetpp.org/. 